\title{
"L'enfant acteur et auteur de santé publique, une recherche-action en classe maternelle à Herchies ${ }^{1}{ }^{1}$
}

\author{
Olivier Francomme
}

\begin{abstract}
Résumé

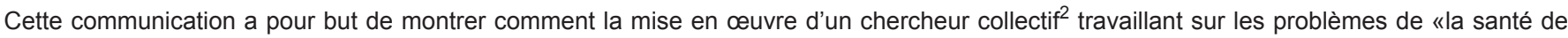
l'écolier» a amélioré de manière sensible les conditions de vie et la sécurité des enfants dans une école maternelle. L'implication des enfants dans le processus tâtonnant d'une recherche-action a permis l'éveil de la conscience aux problèmes de santé. La forme coopérative instituée depuis quelques années dans l'école et la classe maternelle en particulier, a permis une appropriation « naturelle » par les enfants d'un domaine qui leur est sensible. De plus, ce travail a contribué de façon indissociable et importante à la formation de tous les membres de la communauté scolaire, éducative, débordant du strict cadre scolaire mais s'inscrivant avant l'heure dans les nouveaux programmes de l'éducation nationale. Nombre de problèmes de santé abordés dans le cadre de cette recherche, ont été initiés par les enfants, les enseignants et les parents. La recherche d'une continuité éducative a été constante pour la pertinence de ce travail qui n'avait de sens qu'en s'inscrivant dans la continuité des espaces de vie des enfants: dans l'école mais aussi dans la famille, et les autres espaces sociaux.
\end{abstract}

\section{"A criança atora e autora de saúde pública, uma pesquisa-ação em classe maternal em Herchies"}

\section{Resumo}

Esta comunicação tem como objetivo mostrar como o trabalho de um pesquisador coletivo ${ }^{3}$, trabalhando com problemas de "saúde do aluno", melhorou de maneira sensível as condições de vida e a segurança das crianças em uma escola maternal. A implicação das crianças no processo inicial de uma pesquisa-ação permitiu o despertar de consciência para os problemas de saúde. A forma cooperativa instituída depois de alguns anos na escola e na classe maternal em particular, permitiu uma apropriação "natural" pelas crianças de um domínio que Ihes é sensível. Além disso, esse trabalho contribuiu de maneira indissociável e importante na formação de todos os membros da comunidade escolar, educativa, ultrapassando o estrito quadro escolar, mas inscrevendo-se prematuramente nos novos programas da educação nacional. Inúmeros problemas de saúde abordados no quadro desta pesquisa foram iniciados pelas crianças, pelos professores e pelos pais. A procura de uma continuidade educativa foi constante para a pertinência desse trabalho que tinha sentido apenas se se inscrevesse na continuidade dos espaços de vida das crianças: na escola, mas também na família, e nos outros espaços sociais.

\section{El niño actor y autor de salud pública: una investigación-acción en aula maternal en Herchies}

\footnotetext{
Resumen

Esta comunicación tiene como objetivo mostrar cómo el trabajo de un investigador colectivo ${ }^{4}$, trabajando con problemas de "salud del alumno", mejoró de manera sensible las condiciones de vida y la seguridad de los niños en una escuela infantil. La participación de los niños en el proceso inicial de una investigación-acción permitió el despertar de la conciencia para los problemas de salud. La forma cooperativa instituida después de algunos años en la escuela, y en el aula maternal en particular, permitió una apropiación "natural" por parte de los niños de un dominio que les es sensible. Además, este trabajo contribuyó de manera indisociable e importante en la formación de todos los miembros de la comunidad escolar educativa, más allá del estricto cuadro escolar, introduciéndose prematuramente en los nuevos programas de la educación nacional. Innúmeros problemas de salud tratados en el marco de esta investigación fueron iniciativas de los niños, de los profesores y de los padres. La busca de continuidad educativa fue constante para la pertinencia de este trabajo que sólo tendría sentido si se inscribiera en la continuidad de los espacios de vida de los niños: en la escuela, pero también en la familia y en otros espacios sociales.

1 Texte de communication Olivier Francomme mars 2010 colloque international « L'école maternelle : une école de demain »

2 Ce terme sera défini plus précisément dans la suite de l'article.

3 Este termo será definido mais especificamente na sequência do artigo.

4 Esta palabra será definida más especificamente en el artículo.
} 


\title{
The child actor and author of Public Health, an action research \\ in maternal class at Herchies
}

\begin{abstract}
Abstrat
In this study we aim at showing how the collective work of a researcher, working with issues of "health of the student," improves the living conditions and safety of children in a nursery school. The involvement of children in the initial process of an action research allowed the awareness to health problems. The cooperative form instituted after a few years in school and nursery class in particular, allowed a "natural" appropriation by the children of a domain that is sensitive to them. We aim at contributing to the education of all members of the school community, educational community, going beyond the strict framework, by inserting new national education programs. Numerous health problems addressed in this work were started by children, teachers and parents. The demand for continuing education has been a constant for the relevance of this work . It only makes sense if it inscribes in the living spaces of the children in school, in families, and other social spaces.
\end{abstract}

\section{Introduction}

\section{l'école et la santé de l'écolier}

La santé de l'écolier relève de nombreuses dimensions et de nombreux dispositifs dans le parcours scolaire de l'enfant, ce qui montre la préoccupation constante de toutes les dimensions du bien-être de l'enfant dès son plus jeune âge dans l'école française.

D'un côté, la santé de l'enfant relève de comportements, d'habitudes, d'apprentissages, mais elle relève aussi d'une nécessaire prévention afin de ne pas se mettre en danger, ou de ne pas dégrader, même de manière discrète, ce qui constitue chez eux un véritable capital santé.

D'un autre côté, l'institution a pris en charge, sous de nombreuses formes, la santé des enfants qui nous sont confiés : par des visites médicales régulières et obligatoires, par des éléments des programmes officiels de l'Education nationale ${ }^{5}$, et par des dispositifs permettant de faire appel à un certain nombre de professionnels en cas de troubles avérés ou de suspicion.

De plus, la circulaire du BO n³3 du 14 septembre 2006 sur l'éducation à la responsabilité en milieu scolaire complète même cette approche en affirmant « l'importance accordée à l'acquisition, par ces mêmes élèves, de savoirs et de comportements nécessaires pour prévenir une situation de danger, se protéger et porter secours. " Cette éducation a pour vocation, en dépassant le cadre scolaire, « d'affirmer la place du citoyen au cœur de la sécurité civile. "

Pour l'école maternelle, la mise en œuvre de ce programme « apprendre à porter secours » demande de " repérer une situation inhabituelle ou de danger, demander de l'aide pour être secouru ou porter secours".

Le socle commun des savoirs indispensables s'inscrit de plus dans une harmonisation européenne ${ }^{6}$ qui nous donne l'occasion d'aller voir ce qui se passe ailleurs.

5 Voir en annexe une liste des principaux textes officiels. 6 Décret du 11 juillet 2006 relatif au SOCLE COMMUN DE CONNAISSANCES ET DE COMPÉTENCES.
Mais, il faut le rappeler, le domaine particulier de la santé ne peut limiter son champ d'action à l'école. Cette préoccupation étant aussi constante chez les parents, il nous était évident qu'en les associant nous augmenterions considérablement l'impact du travail à mener.

\section{Cadre général de la recherche-action}

La classe maternelle d'Herchies est une classe multiâge qui regroupe tous les enfants du cycle 1 du village, soit une petite trentaine d'enfants de 3 à 5 ans.

Etant titulaire depuis plusieurs années sur le poste, la classe fonctionnait en classe coopérative en pédagogie Freinet, dont j'étais membre du groupe départemental depuis mon entrée à l'Ecole Normale d'instituteurs, ainsi que responsable de publication du bulletin départemental «Sur la Brèche ».

La spécificité de cette forme pédagogique ${ }^{7}$ attribue à l'enfant le pouvoir d'intervenir sur les principaux aspects de sa vie scolaire, dans la gestion du temps, de l'espace et des apprentissages ${ }^{8}$.

Les apprentissages sont eux-mêmes nourris des pratiques d'expression libre, de tâtonnement expérimental et de toutes formes de médiation des langages. Les enfants avaient à disposition les outils et tout le matérialisme de cette pédagogie fondamentalement praxique, entendue au sens de Francis Imbert (1985)

Un des intérêts de cette organisation, c'est la circulation de la parole, la qualité de l'écoute et de l'expression des enfants $^{9}$. Parmi les lieux et moments institués pour la parole, il y avait le conseil, mais il avait été revêtu ici d'une forme particulière: bien sûr nous avions gardé un véritable conseil

7 La forme spécifique de la pédagogie Freinet a été abordée dans un article publié au Brésil. Francomme Olivier, (2010), et dans un ouvrage de Louis Henri Go. Go Henri Louis, (2007)

8 Pour la description générale des techniques et des outils de la pédagogie Freinet, vous trouverez en annexe les différents sites où sont archivés les témoignages de pratiques usuelles dans l'ICEM, et de ma classe en particulier.

9 Cette prise en compte de la parole de l'enfant est une première phase de reconnaissance au sens développé par Hervé Cellier, (2010), dans son livre sur la démocratie d'apprentissage. 
de coopérative hebdomadaire, le samedi matin, qui nous permettait de revenir sur des événements de la semaine " à froid », mais en plus, j'avais ajouté en fin de journée un moment de conseil quotidien, mieux adapté aux capacités mnémoniques des enfants. L'entretien du matin était lui aussi propice à la réflexion.

En classe, parmi les principaux intérêts de l'institutionnalisation de la parole et de sa différenciation selon les lieux, c'est de permettre l'émergence d'une réflexion collective à laquelle chacun contribue, et où peu en échappent. Les moments très courts de juxtaposition des préoccupations individuelles deviennent au fil de l'année, des années, un véritable lieu d'échanges et de contributions. Ces moments étaient souvent animés par un président de séance, me réservant le rôle de secrétaire qu'ils ne pouvaient pas assumer.

Dans la classe maternelle d'Herchies, beaucoup d'autres personnes contribuaient à son fonctionnement : c'étaient les parents d'élèves et toutes sortes d'intervenants sollicités selon les besoins soulevés par l'activité de la classe. La classe maternelle d'Herchies, dans son environnement, est devenue naturellement le laboratoire de l'expérimentation, puis de la recherche. La place des enfants y a été prépondérante après qu'ils ont été sollicités tant pour la mise en relief des problèmes de santé, que pour la mise en place des propositions de travail.

Le Groupe départemental de l'École Moderne Pédagogie Freinet de l'Oise (IDEM 60), associé à celui de la Somme, a été un des lieux de réflexion, celui de l'articulation générale de la recherche. Au cours des réunions mensuelles de travail, il y avait régulièrement des comptes rendus des étapes, et des projets de communication au groupe ${ }^{10}$. Ce fut aussi le moment de rencontre et d'échanges avec un certain nombre d'invités : médecins, orthophoniste,... II a constitué un des réseaux de discussion et de réflexion majeur. C'est l'un des cercles du chercheur collectif peu à peu constitué.

Pour le côté "Santé », il s'est agi de travailler avec un certain nombre d'interlocuteurs : des médecins et autres professionnels de santé, des structures comme le GRASB ${ }^{11}$, ou l'Ecole nationale de santé publique de Rennes (avec Jacques Chaperon). Les modalités de travail de ce second réseau de réflexion ont été différentes, de par l'éloignement et les contraintes professionnelles qui leur étaient spécifiques. Nous nous sommes rencontrés plus longuement (rencontres de plusieurs jours) mais à des dates plus espacées.

Les rencontres avec les parents d'élèves ont été un autre lieu de laboratoire, parce que les parents étaient les premiers concernés par les dispositifs éducatifs et pédagogiques mis en place pour lesquels leur complicité était nécessaire.

Tous ces réseaux de travail constituent l'ossature et la matrice d'un chercheur collectif ${ }^{12}$ qui a œuvré pendant une dizaine d'années. Le moteur de ce réseau a été la forma-

10 Voir en annexe une liste partielle des publications.

11 GRASB : Groupe de recherche action sur la santé en Bourgogne. 12 D'autres chercheurs collectifs ont été analysés dans les pratiques scientifiques de l'ICEM, tel celui mis en œuvre autour de Pierre Guérin dans les pratiques audiovisuelles, celui autour de Jean le Gal sur l'autogestion, ... tion sur la préoccupation de la santé des jeunes. Le cadre de référence théorique a été constitué par la nécessaire confrontation interdisciplinaire, quand les sciences de l'éducation doivent cohabiter avec la médecine, mais aussi avec l'éthique et la philosophie.

Le chercheur collectif relève d'une organisation différente de la recherche, qui a été décrite, envisagée, mis en œuvre de manières différentes au cours de l'histoire. C'est René Barbier (1996) qui a le mieux contribué au développement de ce concept, dans son ouvrage sur la rechercheaction : "Le chercheur collectif est un groupe sujet de recherche constitué par des chercheurs professionnels (venant d'organismes de recherche ou d'universités) et des membres à part entière (mais particulièrement impliqués), de la population concernée par l'enquête participative. ».

Historiquement c'est sans doute Antonio Gramsci, écrivain et théoricien politique italien marxiste, qui, au travers "des intellectuels organiques ${ }^{13}$, a tenté de promouvoir l'appropriation de la culture dans toutes les classes sociales (et particulièrement les classes défavorisées). II a crée une rupture épistémologique dans la science en tentant de rompre la césure culturelle et sociale des sciences.

Bruno Latour (2005) propose de son côté « le parlement des choses ", comme dispositif de confrontation entre la politique, l'administration et la science.

Un chercheur collectif poursuit plusieurs ambitions: de la formation, de la production de savoir, et une jubilation collective (donner du sens professionnel).

Un chercheur collectif fonctionne de plusieurs manières: par coopération, par formation, par accompagnement, par confrontation,... par tâtonnement expérimental.

Le chercheur collectif ne peut être constitué que progressivement, et il n'est que l'aboutissement d'un long cheminement dans la structuration d'un vaste espace de recherche dont les différents pôles (ou réseaux) travaillent de manière autonome.

Le fonctionnement du chercheur collectif sur la santé de l'écolier concernait 4 réseaux de travail ayant chacun leurs règles propres de fonctionnement:

-l'école, la classe, les enfants, les personnels éducatifs (bilans, conseils) ;

-les médecins, les enseignants (rencontres à thèmes, correspondance);

-le conseil d'école, les conseils de classe (ordre du jour, publication du bulletin « le Lien »);

-le groupe Freinet départemental, régional (réunions pédagogiques, publications Sur la Brèche).

Ces réseaux peuvent travailler en transparence ou non (nécessité du secret professionnel, médical ou éducatif),

13 Ces intellectuels sont conçus organiquement par les classes sociales. Ils s'opposent aux intellectuels de l'intelligentsia qui se considèrent comme une classe à part. 
il existe des temps possibles de contributions ponctuelles d'un réseau à l'autre.

\section{Le projet sur la santé de l'écolier}

Au tout départ, c'est en tentant de répondre à une série de questions dans l'école, que nous avons commencé un travail de formation, qui peu à peu est devenu un véritable travail de recherche-action. Notre questionnement portait sur la problématique de la santé :

- Quelles sont les différentes pathologies médicales qui peuvent affecter, même temporairement, les apprentissages scolaires?

- Quelles en sont les manifestations, et comment les diagnostiquer afin de les prendre en compte dans un parcours éducatif et de santé global ?

- Quels outils peuvent être mis à la disposition de tous les membres du collectif éducatif : enseignants, parents, médecins scolaires, mais aussi des enfants eux-mêmes ? Quelles compétences pour chacun, quelles responsabilités?

Cette première série de questions nous a permis de travailler et de réfléchir sur « les troubles discrets de la perception sensorielle », des pratiques corporelles novatrices: " la gymnastique dans l'espace », la nutrition, la connaissance du système de santé publique, ....

Aujourd'hui, les instructions officielles confirment la volonté de "donner du sens à la culture scolaire ${ }^{14}$ ", en proposant de développer « les compétences sociales et civiques dans l'institution scolaire, ainsi que l'autonomie et l'initiative des élèves ", et ce, dès l'école maternelle.

Bon nombre de domaines du socle commun auront été abordés tout au long de ce travail mené en classe, qu'il s'agisse de l'expression orale, le vocabulaire, l'utilisation de la langue comme instrument de pensée, la justesse dans le propos, le dialogue, une entrée dans la culture scientifique, l'approche concrète, scientifique, l'organisation et la gestion de données, le raisonnement logique, la déduction, l'observation, l'esprit critique, l'observation de règles, la responsabilité face à la santé, et bien sûr un certain nombre de connaissances dans différents champs disciplinaires, des sciences du vivant au français.

Bien sûr, les compétences sociales et civiques ont été particulièrement abordées au cours de cette recherche, qu'il s'agisse du vivre en société, de se préparer à la vie de citoyen, ou dans l'autonomie et l'initiative des enfants.

II faut considérer ces compétences de manière interdisciplinaire, voire transdisciplinaire, et l'école maternelle est sans doute l'endroit le plus adapté dans sa forme pédagogique pour initier les enfants à ces compétences fondamentales. L'ombre portée des compétences scolaires doit

14 Ibid Décret JO du 11 juillet 2006. à présent franchir le cadre de l'école, et s'inscrire dans la perspective de la vie en société.

Mais les ambitions des instructions officielles vont plus loin en soulignant l'importance de la mobilisation de tous les acteurs de la communauté scolaire dans des partenariats portant sur la protection du milieu scolaire ${ }^{15}$. Même si ce décret s'adresse principalement aux établissements du second degré, il est clairement indiqué qu'il s'adresse à tous les niveaux de la scolarité.

Curieusement, cette expérience a anticipé la loi, principalement quand elle met en avant l'importance du diagnostic de sécurité. De même, l'institution d'espaces de confrontation avec les parents (par les réunions et au travers du "Lien ») a contribué à leur formation ainsi que celle de tous les membres de la communauté éducative.

\section{L'éducation au risque, le brevet de secourisme en maternelle ${ }^{16}$.}

Nous nous arrêterons plus longuement sur ce thème qui a synthétisé le plus d'éléments plausibles dans la voie de la définition d'une nouvelle épistémologie de la démarche scientifique, voire de la recherche.

Dans la liste des préoccupations des enseignants sur les problèmes de santé et de sécurité physique des jeunes qui nous sont confiés, la cour de récréation demeure un véritable lieu de tous les dangers. II y a pas mal de littérature sur le sujet, mais peu de prise en compte générale dans la gestion des risques et son adaptation à tous les cas particuliers de chaque espace récréatif.

Au cours des discussions avec les médecins une idée a germé : «Après tout, la cour de récréation, c'est leur problème à eux (les enfants) de gérer cela ! ». Cette réflexion a donné lieu à une véritable prise en charge progressive de cette préoccupation en réunion de bilan ou de conseil, de la classe. Très vite les enfants ont proposé de prendre eux-même en charge les soins, mais il était évident qu'ils ne pouvaient pas faire n'importe quoi.

Dans la classe Freinet, le brevet ${ }^{17}$ a une place particulière, il atteste d'un ensemble cohérent de compétences qui permet d'accéder à un certain niveau de responsabilité, qui donne droit à l'exercice d'un métier, ou de la prise en charge d'une tâche.

Définir le brevet de secourisme a donc été facilité, il correspondait, par simple transfert, à un nouvel exercice de la responsabilité, comme l'instituaient déjà les « métiers de la classe » : jardinier (responsable des plantes de la classe), documentaliste (responsable de la bibliothèque), responsable de l'heure, ... Obtenir le brevet pour les enfants, c'était être capable de lister tous les éléments d'un processus de soin, et de satisfaire à sa passation de manière pratique.

15 Décret JO du 30 novembre 2006.

16 Ce témoignage a fait l'objet d'un dossier spécial de la revue départementale de l'IDEM 60 "Sur la Brèche », de plus cela a été le thème d'un dossier / mémoire dans le cadre du concours interne des professeurs des écoles.

17 Freinet C, (1949). 
Mais cette discussion s'est déroulée dans plusieurs espaces du chercheur collectif, donc avec les médecins scolaires et les parents, afin de bien cerner tous les enjeux et toutes les contraintes de la mise en œuvre de ce brevet.

Parmi les éléments de la réflexion, initiée dans tous les lieux du chercheur collectif, il y a eu :

-définir les limites de l'intervention : déterminer ce qui relève du simple bobo et ce qui nécessite un examen approfondi ;

-garantir la protection des secouristes : contagions ... ;

-effectuer les soins (rassurer, nettoyage, protection,...) ;

-remplir et comprendre l'importance du cahier d'infirmerie.

Nous avons rapproché deux techniques investigatrices : une, empirique qualitative, dans le domaine épidémiologique (par le biais d'une enquête sur les incidents et accidents dans la cour) et une autre, participative (en associant les enfants à la gestion et l'analyse des soins infirmiers).

D'un côté, sous couvert éducatif et pédagogique de la nécessité de confier des responsabilités en matière de santé, il a été établi des protocoles de soins à appliquer par des secouristes en herbe, sur les enfants qui se faisaient « des petits bobos » dans la cour de récréation. D'un autre côté, l'analyse du cahier d'infirmerie a permis de mettre en évidence, certaines caractéristiques répétitives de l'accidentologie.

L'obtention des diplômes de secourisme était très rigoureuse et de plus interdisait l'intervention d'un secouriste isolé (nécessité d'une grande clarté dans l'administration des soins). Mais malgré de grandes exigences, bon nombre de jeunes ont passé brillamment le brevet (il y a même eu 2 brevets différents : niveau 1 et 2 ) et ils ont pu exercer leurs compétences avec une grande fierté dans la cour de récréation, voire sur tout le temps scolaire.

Parmi les obligations du secouriste, le rapport d'incident était obligatoire, donc après chaque intervention, il fallait remplir « le cahier d'infirmerie ${ }^{18}$ ce qui en maternelle ne pouvait se faire sans l'aide d'un adulte ou d'un autre enfant " écrivain ». C'était la base de l'autre objet de la démarche, l'enquête épidémiologique.

\section{Quelques éléments saillants de ce travail :}

Aspects positifs

-la mise en œuvre du brevet de secourisme a été facile, parce qu'elle s'inscrivait dans le cadre du fonctionnement global de l'école. On a réalisé une trousse à pharmacie adaptée à l'usage spécifique des secouristes ;

18 Ce cahier était directement issu des centres de loisirs, sans modification. -des liens ont été créés entre enfants : il est arrivé que des petits ont soigné des grands... et les liens ont perduré après ;

-il y a eu une véritable évolution dans l'appropriation de la cour : la nécessaire fragmentation de l'espace, et la création de zones réservées à certaines activités ;

-la prise en compte de l'intégrité corporelle de l'autre, le rapport à l'altérité des enfants a évolué : tomber est souvent fortuit, mais la prise en compte de la douleur de l'autre ;

-l'espace de réflexion autour des accidents de la cour de récréation a débouché sur des réflexions très importantes et variées qui a impliqué de nombreux acteurs (aménagements de la cour de récréation, élargissement à d'autres aspects de la santé,...).

\section{Aspects négatifs}

-tous les parents n'ont pas partagé a priori l'enthousiasme d'une telle pratique, en particulier dans le rapport " au sang » (problème religieux pour certains). De plus, l'épidémie du SIDA a quelque peu modifié les protocoles, et rendu difficilement opérables certaines pratiques dont l'intervention sur des blessures, si modestes soient-elles;

-tous les enfants ne se sont pas inscrits dans la démarche de devenir secouriste, même s'il pouvait dépasser le cadre de la cour de récréation ;

-le brevet de secourisme est quasiment tombé quelques mois après sa création, faute de victimes !

Aspects en question :

-il est difficile d'attribuer à ce brevet de secourisme l'entière responsabilité de l'amélioration du cadre de la récréation, d'autres pratiques ont sans doute contribué : les ateliers décloisonnés par exemple (qui ont aussi facilité le dialogue entre petits et grands).

Dans les faits, en très peu de temps, il n'y a plus eu d'incidents dans la cour de récréation, même les bagarres se sont considérablement réduites, et il y avait très peu d'égratignures... Une majorité d'enfants avait souhaité passer leur diplôme de secouriste $\left(1^{\circ}\right.$ et $2^{\circ}$ niveau) et la cour de récréation s'était considérablement calmée. De plus les élèves des autres classes avaient souhaité participer à cette démarche, nous allions vers une généralisation.

\section{Nous avons pu avancer quelques conclusions:}

-la prise en compte de l'autre : les enfants ont développé une attitude d'ouverture et de prise en compte 
de l'existence de l'autre, dans son humanité. Rassurer un autre enfant qui pleure, s'attacher à l'expression de sa douleur, lui apporter des soins, ... a provoqué une véritable prise en compte de l'altérité ;

-la nécessité de préserver des espaces pour chacun, chaque activité selon des prérogatives implicites ou explicites a contribué à la diminution des heurts, et au respect de chacun et de ses besoins kinesthésiques ;

-la prise en compte du risque dans les activités et son encadrement par tous se sont avérés de puissants outils éducatifs et de prévention des accidents (en particulier par la diminution des attitudes et des actes accidentogènes).

Mais d'autres éléments doivent être pris en compte :

Au moment de la consultation mensuelle du cahier d'infirmerie en classe, nous avons pu clairement identifier : les lieux accidentogènes, les moments propices aux incidents, les types de pathologies traitées.

Sur les lieux, il est apparu évident que certains endroits nécessitaient de règles (ne pas courir à certains endroits, comme ne pas jouer aux billes sous la cage à écureuil,...), mais par l'examen du cahier d'infirmerie, elles sont devenues évidentes et naturelles donc n'ont pas eu à s'imposer artificiellement.

Sur les moments : à ce moment là, les début et fin de récréation s'avéraient les moments les plus propices aux incidents. Nous avons trouvé ensemble des procédures facilitant le démarrage des récréations et le retour au calme progressif (entrées et sorties échelonnées par exemple, distribution et rangement des accessoires, large utilisation de tous les espaces disponibles, ...).

Sur les types de pathologies: hormis les bosses et égratignures, la consultation du cahier d'infirmerie a permis de parler des certaines affections (fièvres, maux de ventre, verrues plantaires, ...) en établissant un espace de discussion autour de la santé : doit-elle être contenue à l'espace privé ? (c'est récent en France, et ce n'est pas un modèle universel) Quelle peut être la part de chacun dans le processus de soin ? (part des spécialistes, de la réflexion sociale?)

Les parents ont toujours été informés des démarches en cours, et de l'accompagnement (rassurant) d'une équipe médicale. Les réunions de parents d'élèves ont permis d'expliquer la pertinence des expériences que nous mettions en place, et de désamorcer les inquiétudes légitimes de chacun.

Un acte éducatif simple : mise à disposition de 2 outils à des enfants (carnet d'infirmerie et brevet de secourisme) a permis de mener les prémisses d'une véritable recherche en relation à une problématique de santé communautaire :

-démarche investigatrice (action, recueil de données) ;

-analyse des données / résultats (localisation, sériation) ; -appropriation, mise en relief des causes, des sources d'atteinte à l'intégrité, aux dommages corporels ;

-évaluation, mesure de l'impact de la mise en place d'outils expérimentaux.

\section{L'enfant acteur de santé publique ou communautaire.}

Ce long travail a permis de mettre en évidence l'intérêt de ce type de démarche associant toutes sortes de parties, en respectant leurs spécificités : des enfants, des parents, des enseignants, des médecins.

Un certain nombre de prérogatives scientifiques ont été respectées et ont abouti à une véritable co-formation scientifique dont toutes les parties ont été bénéficiaires.

II y a eu de nombreux moments de discussion et d'échange intenses sur la prise en compte de la santé au moins au niveau communautaire :

- Comment prendre en compte le corps, comment toucher, agir sur le corps ?

- Qu'est-ce qui relève du secret médical ?

- Qu'est-ce que recouvre l'obligation de réserve en matière de santé ?

Au bilan de ce travail, et pour résumer la nature des différentes actions entreprises, nous pouvons mettre :

- Des publications pédagogiques et médicales conséquentes.

- Sur les pratiques mises en œuvre

.L'observation clinique

.L'approche empirique qualitative dans le domaine épidémiologique

.Le travail interdisciplinaire

.La nécessaire coopération entre tous les professionnels concernés par un phénomène complexe.

- Sur l'impact de ce long moment de travail :

-inscription de la santé dans une démarche globale (modifier des comportements) ;

-mise en place d'un dispositif de recherche original, performant et adaptable ;

-socialisation importante des résultats : enfants, parents, enseignants, professionnels de santé, collectivités locales, ...

En plus du journal d'école, un journal de liaison des adultes a vu le jour : "Le Lien ». Ce bulletin permettait de poursuivre les discussions entamées dans les réunions de 
la classe et l'école. Par exemple, il a été le lieu de publication d'un cadre de référence théorique. II permettait aussi de " vulgariser " les propos des spécialistes, et de démarrer des discussions sur des thèmes à venir.

Encore une fois, dans cette démarche longue, nous avons mis en œuvre une autre manière de concevoir notre relation aux autres et aux connaissances. Nous avons fait avancer collectivement un groupe important et varié de personnes dans la réflexion et dans la prise en charge de son parcours de formation.

C'est une autre manière de concevoir une éthique scientifique et une épistémologie de la recherche.

\section{Essais de schématisation}

Schéma interdisciplinaire 1- Organisation de la recherche, espaces du chercheur collectif

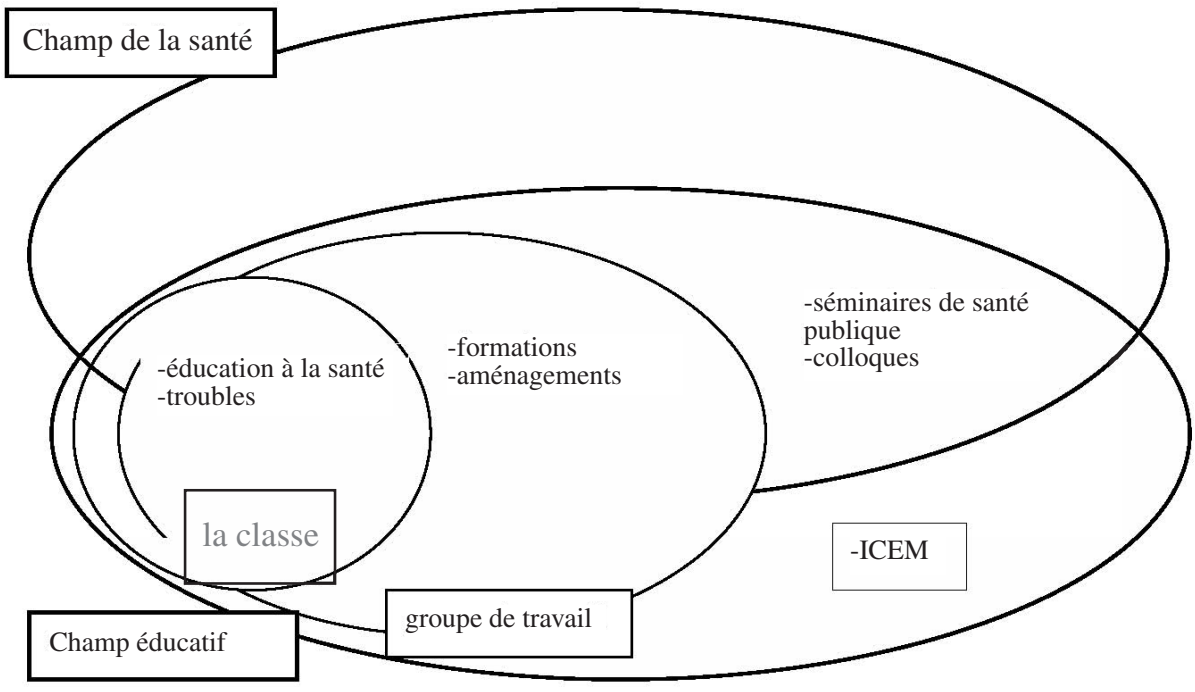

Schéma interdisciplinaire 2 - les productions scientifiques, professionnelles

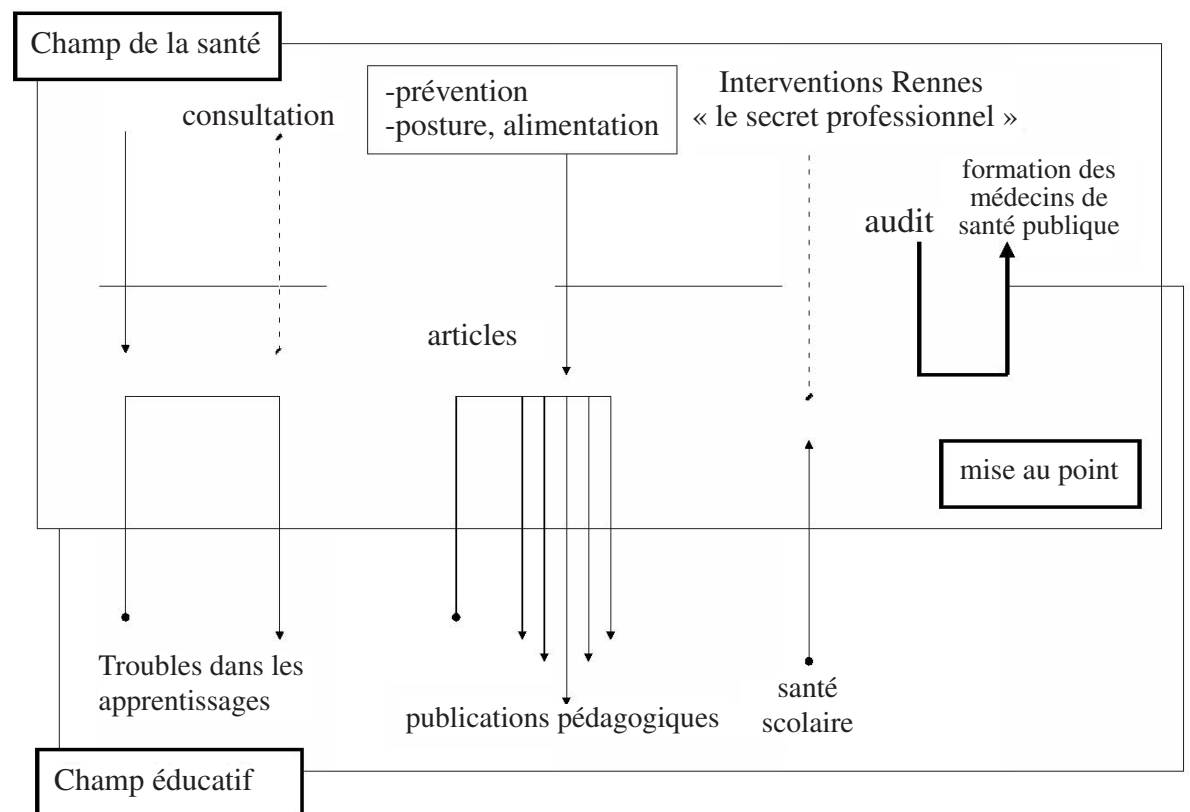




\section{Bibliographie}

Barbier, R. (1996). La recherche action. Paris: Anthropos.

Cellier, H. (2010). La démocratie d'apprentissage. Paris : I'Haramattan.

Francomme, O. (2010). La Forme Scolaire et la Pédagogie Freinet, à l'Épreuve de l'Éducation Comparée. Sur le livre d'Yves Reuter et autres études longitudinales. Educação: Teoria e Prática, 20(34), 13-33.

Francomme, O. Notes de lecture sur l'ouvrage de Jean le $\mathrm{Gal}$ : Le maître qui apprenait aux enfants à grandir. Un parcours en pédagogie Freinet vers l'autogestion. Revue Les amis de Feinet, Mayenne, à paraître.

Francomme, O. (2009). Notes de lecture sur le livre de Gast M, Guérin $M$ et $C$, Pierre Guérin, Sur les pas de Freinet. Revue Les amis de Freinet, Mayenne.

Freinet, C. (1949). Brevets et Chefs d'œuvre. Cannes : CEL.

Louis G. H. (2007). Freinet à Vence : Vers une reconstruction de la forme scolaire. Rennes, França : Éditions PUR.

Imbert, F. (1985). Pour une praxis pédagogique. Paris: Matrice.

Latour, B. (2005). Nous n'avons jamais été modernes. Essai d'anthropologie symétrique. Paris : La Découverte.

\section{Textes officiels : Bulletin officiel (B.O.)}

Circulaire BO $\mathrm{n}^{\circ} 10$ de 9/03. (2006). Scolarisation des élèves handicapés - Parcours de formation des élèves présentant un handicap. Un projet personnalisé de scolarisation définit les modalités de déroulement de la scolarité et les actions pédagogiques, psychologiques, éducatives, sociales, médicales et paramédicales répondant aux besoins particuliers des élèves présentant un handicap.

Circulaire BO n`13 31/03. (2006). Rentrée Scolaire. Préparation de la rentrée 2006 (dont l'affirmation du droit à la scolarité pour les élèves handicapés).

Circulaire BO $\mathrm{n}^{\circ} 30$ du 27/07 (2006). Éducation à la sécurité. Sensibilisation à la prévention des risques, aux missions des services de secours, formation aux premiers secours et enseignement des règles générales de sécurité (Cette circulaire précise les conditions de mise en oeuvre de cette sensibilisation dans une démarche d'éducation à la responsabilité en milieu scolaire.).
Circulaire BO n`31 du 31/08 (2006) Lutte contrela violence. Prévention et lutte contre la violence en milieu scolaire (L'acte pédagogique et le cadre éducatif sont les premiers socles de la lutte contre la violence scolaire. Mais l'action éducative doit être complétée par des mesures d'accompagnement et de prévention qui ne sauraient exclure le recours à la sanction.)

Circulaire BO n³3 du 14/09 (2006). Éducation à la responsabilité en milieu scolarie. Rappel du dispositif législatif et réglementaire et modalités de mise en oeuvre de cette sensibilisation à la prévention des risques et formation aux premiers secours. Inscription dans les projets et la vie scolaire des établissements, implication des comités et instances représentatives, organisation des dispositifs de pilotage. (sensibilisation à la prévention des risques, aux missions des services de secours, formation aux premiers secours. Cette circulaire précise les conditions de mise en oeuvre de cette sensibilisation dans une démarche d'éducation à la responsabilité en milieu scolaire.)

Décret JO du 11 juillet (2006). Relatif au Socle commun de connaissances et de compétences.

Décret JO du 30 novembre (2006). Relatif à la protection du milieu scolaire.

\section{Sites électroniques :}

ICEM : http://www.icem-pedagogie-freinet.org/

IDEM 60/80 : http://idem6080.lautre.net/

Les amis de Freinet : http://www.amisdefreinet.org/

-Sur les brevets : http://www.freinet.org/icem/archives/benp/benp-42/ benp42.htm

\section{Bibliographie : dossier : « la santé de l'écolier »}

Articles publiés régulièrement dans la revue départementale de I'IDEM 60 « Sur la Brêche » :

-Fortin Jacques, Les postures de l'écolier, 11p.

-Francomme Olivier, Questionnaire (de rentrée) aux familles, 2p.

-Francomme Olivier, Le sucre, 2p.

-Francomme Olivier, Excès de viande ?, 2p. 
-Francomme Olivier, L'enfant, le sommeil et l'école, Notes de lecture sur le livre d'Hubert Montagner Les rythmes de vie de l'enfant et de l'adolescent. , $5 p$.

-Francomme Olivier, Un brevet de secourisme à la maternelle, 1987, $2 p$.

-Francomme Olivier, L'enfant acteur de santé. Education au risque, réduction des risques., Notes de lecture sur les ouvrages de Fortin Jacques et Devienne B., $5 p$.

-Francomme Olivier, L'image inconsciente du corps, Notes de lecture sur le livre de Françoise Dolto, $1 p$.

-Francomme Olivier, Organisation générale de la santé scolaire, 1p.

-Francomme Olivier, 5 gestes qui sauvent, Notes de lecture revue La santé de l'écolier $n^{\circ} 114,2 p$.

-Francomme Olivier, Les gestes qui sauvent, Notes de lecture revue La santé de l'écolier $n^{\circ}, 2 \mathrm{p}$.

-Francomme Olivier, Les empoisonnements de l'enfant par ingestion, Notes de lecture revue La santé de l'écolier n¹18, 2p.

-Francomme Olivier, Une trousse à pharmacie homéopathique, 1p.

-Francomme Olivier, Test de vision de loin pour lecteurs et non lecteurs, $7 p$.

-Francomme Olivier, Matériel et protocole d'une épreuve de dépistage (de la surdité) chez les enfants de 4 à 7 ans, $5 p$.

-Gerbeau Claudine, Compte rendu de la recherche effectuée dans le département de la Dordogne sur le mobilier scolaire, $6 \mathrm{p}$.

-Bulletin Officiel de l'éducation nationale, Accidents alimentaires collectifs en milieu scolaire, BO n40 du 9 novembre 1989.

-Bulletin Officiel de l'éducation nationale, Lutte contre le tabagisme dans les établissements publics locaux d'enseignement, BO n40 du 9 novembre 1989.

-Bulletin Officiel de l'éducation nationale, Surveillance et sécurité des élèves des écoles élémentaires et maternelles, BO n46 du 21 décembre 1989.

-Bulletin Officiel de l'éducation nationale, Durées et conditions d'éviction, mesures de prophylaxie à prendre à l'égard des élèves et du personnel dans les établissements d'enseignement et d'éducation publics et privés en cas de maladies contagieuses. BO n`8 du 22 février 1990.

\section{Dossiers :}

-Premier dossier récapitulatif : « La santé de l'écolier »

-«Aménager la cour de récréation »

-« La gymnastique aérienne »

-«Les spécialistes autour de la maternelle »

\section{Autres publications :}

-Francomme Olivier, Le brevet de secourisme à la maternelle, Dossier de concours de recrutement interne des Professeurs des écoles, Sur la Brèche,

Documents :

\section{Le cahier d'infirmerie :}

Chaque ligne comporte un certain nombre de renseignements à compléter :

-date et heure de l'incident

-lieu de l'incident

-nature des dommages

-remèdes apportés

-remarques

\section{Protocole général en cas d'incident}

1- informer les enseignants d'un incident (accident) dans la cour (ou ailleurs)

2- accompagner le camarade blessé à un lieu de soin (près d'un enseignant)

3- prodiguer les soins

4- remplir le cahier d'infirmerie 


\section{Protocole de soin}

1- rassurer le camarade, lui demander s'il veut être soigné ;

2- se protéger, mettre des gants ;

3- durant tout le long des soins, il faut expliquer ce que l'on fait et répondre aux questions ;

4- nettoyer le bobo à l'eau et au savon en utilisant des compresses ;
5- utiliser un cicatrisant si nécessaire ;

6- s'occuper des vêtements (protéger, nettoyer si nécessaire);

7- remplir le cahier d'infirmerie (avec le camarade);

8- accompagner le camarade dans la cour, ou dans un endroit calme, si nécessaire.

Recebido em: 19/04/2011

Aprovado em: 25/06/2011

\section{Sobre a autora}

Olivier Francomme (olivier.francomme@icem-freinet.org)

Formateur premier degré UPJV - IUFM Beauvais

Responsable du secteur international de l'ICEM 